\title{
MODELO DE RESPONSABILIDAD SOCIAL EMPRESARIAL PARA EL MEJORAMIENTO DE COMUNIDADES ASENTADAS EN UN ENTORNO EMPRESARIAL: CASO MUJERES CABEZA DE FAMILIA
}

\author{
RICARDO AUGUSTO PUCHE VILLADIEGO*
}

Recibido 19 de Julio de 2012 / Enviado para Modificación 2 de Septiembre de 2012 / Aceptado 20 de Noviembre de 2012

\section{RESUMEN}

El trabajo nace de la necesidad de las empresas de contar con un programa de responsabilidad social que contribuya con el mejoramiento de su entorno a través de un modelo efectivo que llegue a la comunidad. Para la delimitación del modelo se identificó un grupo de interés seleccionando las comunidades asentadas en el entorno de una unidad empresarial y específicamente mujeres cabezas de familia. Se establece entonces un modelo de responsabilidad social que apunta a mejorar la calidad de vida y el acercamiento de la empresa a la comunidad a partir del sistema de donaciones, donde los recursos económicos (mercancías) se entregan con el objetivo de mejorar el aspecto económico, social y educativo. El modelo diseñado permite un desarrollo social, donde la generación de actividades de Responsabilidad Social Empresarial (RSE) son la base para lograr un bienestar social al grupo objetivo y adicionalmente, se convierte en instrumento para lograr un acercamiento entre la empresa y la comunidad. El modelo opera canalizando mercancías hacia la comunidad, administradas por la empresa, con el propósito de garantizar su correcta disposición.

Palabras clave: Responsabilidad social, grupos de interés, calidad de vida, bienestar, empresa.

\section{ABSTRACT}

The work stems from the need for companies to have a Corporate Social Responsibility program contributes to improving the environment through an effective model that reaches the community. The establishment of the model identified stakeholders by selecting the communities in the environment of a

\footnotetext{
* Magister en Administración de Empresas e Innovación, 2011; Especialista Universitario en Gestión de Empresas; Especialista en Gerencia Financiera; Economista. Investigador del grupo "Democracia y Modernización del Estado Colombiano de la Universidad Simón Bolívar. Asesor. Correo electrónico: ricardopuche@hotmail.com.
} 
business unit, specifically women heads of households. Then established a model of Social Responsibility that aims to improve the quality of life and the company's approach to the community from the donation system, where economic resources (merchandise) are delivered with the aim of improving the economic, social and educational. The model designed allows for social development, where the generation of Corporate Social Responsibility activities (CSR) are the basis for a social welfare in addition to the target, becomes an instrument to achieve a rapprochement between the company and the community. The model operates channeling goods to the community, managed by the company, in order to ensure their proper disposal

Key words: Social Responsibility, Stakeholders, Quality of life, Welfare, Business.

Classification Journal Economic Literature (JEL): M14, I31, D11.

\section{INTRODUCCIÓN}

Este artículo plantea un Modelo de Responsabilidad Social Empresarial para una unidad empresarial dirigido a un grupo de interés, en este caso, la comunidad de mujeres cabeza de familia asentadas en el entorno empresarial, con el fin de ayudar a satisfacer sus necesidades sociales básicas, sirviendo de apoyo en otros aspectos importantes de su vida cotidiana, como el económico y la educación.

Este modelo se centra en los problemas que más aquejan al grupo objeto de estudio, contando además con indicadores que permiten medir el impacto del modelo a partir del desarrollo de la comunidad. Su aplicación de una manera ética y transparente permitirá el fortalecimiento de las relaciones entre el grupo objetivo y la empresa.

Al seleccionar el grupo objetivo se identificó que la jefatura femenina del hogar es un fenómeno creciente en el país y evidencia una transformación de la familia. En Colombia, como en muchos otros países, cada vez más mujeres de todas las condiciones sociales y económicas asumen el rol de proveedoras principales de sus hogares y son reconocidas por los demás miembros como tales. Estas transformaciones en las estructuras familiares y sociales revelan cambios en las relaciones de género y tienen consecuencias directas en el orden económico y en el desarrollo de las sociedades, sobre todo, en zonas de cabecera y, en menor grado, en el resto del país (1). 
La mayoría de estas mujeres no alcanza a devengar un salario mínimo y lo poco que ganan lo tienen que repartir entre un mayor número de dependientes. Sus hogares presentan mayor desnutrición en menores, tasas más altas de trabajo infantil, mas desprotección de los hijos y por lo tanto mayores riesgos de morbilidad comparando con el promedio urbano (2).

De acuerdo con cifras del DANE, se puede apreciar que entre los años 2003 y 2008 aproximadamente 3 de cada 10 hogares a nivel nacional las jefaturas de hogar son femeninas. Análisis previos de los datos del Censo del año 2005 del DANE explican que "las jefaturas de hogar han crecido con relación al número de habitantes. La jefatura femenina registra un leve aumento en los hogares colombianos. En total, el 70\% de las jefaturas de los hogares es ejercida por un hombre y el $29 \%$ por una mujer", lo cual coincide con las cifras de los años 2003 a 2008 (3).

En la búsqueda de un buen diseño de un modelo de gestión en lo concerniente al tema de la responsabilidad social empresarial, donde la empresa genere un beneficio de su entorno y la comunidad en general, se analizaron diferentes modelos de responsabilidad social empresarial aplicados en empresas tales como Bancolombia, Telefónica y Microsoft; esto con el fin de cimentar las bases del modelo propuesto y extractar ante todo los fines y valores propios de un modelo de RSE. Aquellos aplicados en dichas empresas, poseen elementos comunes entre los cuales se puede mencionar el tema de la educación desde distintas perspectivas o enfoques de ayuda, y la sinergia que debe buscarse para que los esfuerzos se encaminen mejor hacia la comunidad y se pueda conocer más a fondo su problemática.

En Colombia se han analizado entre otros, Carvajal y su proyecto ambiental, Microsoft y el impacto de la tecnología en el desarrollo de los pueblos, Bancolombia y su programa de compromiso social en una obligación, Argos y su visión de una empresa que cree en el desarrollo sostenible.

Carvajal, con una serie de proyectos que impactan de manera positiva en la sociedad, la RSE va más allá de su promoción; se trata de la creación de una fundación separada de la empresa a la cual se le destinan recursos cuantiosos para la realización de proyectos de desarrollo para la comunidad. Uno de los ejecutivos de Carvajal, sostiene al respecto: "Las empresas no pueden crecer y desarrollarse, de manera saludable, en una sociedad enferma" (4). 
Como Carvajal, cualquier organización puede ser vista como un conjunto de partes interrelacionadas entre sí y con su entorno, de manera que cualquier variación en alguna de ellas afecta el sistema final. Todas las cosas y entes pueden ser vistas como un sistema (5), por eso la responsabilidad social no es una moda, es una política que más compañías colombianas públicas y privadas, están adoptando dentro de sus prácticas, porque les permite devolver algo a la comunidad donde operan, o incluso a las familias de sus trabajadores con menores ingresos, (6).

\section{DISEÑO METODOLÓGICO DEL MODELO DE RSE}

El diseño del modelo de RSE, se basa en un sistema de donaciones hacia los grupos de interés más cercanos de la empresa a partir de mercancías a disposición. Para el caso de estudio, de estos grupos de interés, se ha seleccionado el de la comunidad que hace parte del entorno y específicamente las mujeres cabeza de familia.

Se selecciona este grupo de mujeres por su alto grado de vulnerabilidad, ya que en su mayoría carecen de recursos económicos, así como el apoyo de un compañero para el sostenimiento de sus hogares. El modelo busca entonces la intervención de aquellas variables que afectan directamente a las mujeres cabeza de familia de la comunidad con el propósito de crear condiciones necesarias para mejorar su calidad de vida, proporcionando un mayor impacto social, así como el acercamiento de la empresa a este grupo.

Entre las variables detectadas se encuentran la dependencia económica, recursos económicos, seguridad social, desempleo, nivel de estudio, sobrecarga laboral, propiedad del bien inmueble y edad de las madres.

Para el desarrollo del Modelo se utilizó el método de investigación deductivo, ya que parte de lo general a lo particular. Su enfoque se fundamenta en lo cuantitativo de las cifras, aunque no lleva hipótesis, por contar con objetivos sólidos que direccionan el curso de la investigación. Adicionalmente, la investigación realizada es de tipo descriptiva ya que conlleva al estudio y análisis de los factores y variables que determinan la situación actual de las personas objeto de estudio.

Para el proceso de elaboración de la muestra no se encontraron datos precisos de la población objetivo, por lo que el criterio de selección se basó 
en la conveniencia, ya que se tuvo que recurrir al edil de la junta de acción comunal y acción social, quienes estimaron un aproximado poblacional de 330. La encuesta se aplicó entonces en una muestra de 43 mujeres cabeza de familia, de las cuales 3 encuestas no se validaron por inconsistencias en el diligenciamiento, obteniendo finalmente 40 encuestas válidas.

En cuanto a los instrumentos utilizados para la investigación se aplicó tanto la observación como la encuesta para la recolección de los datos. Para esta última se emplearon preguntas estructuradas que especifican, en forma previa, tanto el formato como el grupo de alternativa de respuesta.

Se diseñó un cuestionario basado en preguntas socioeconómicas de opción múltiple, las cuales permiten encontrar datos que conlleven a visualizar la situación real de la población objeto de estudio en cuanto a sus condiciones de vida y necesidades tanto fisiológicas como sociales. La encuesta fue realizada en el mes de septiembre de 2010.

La encuesta que se aplicó es de caracterización socioeconómica, teniendo en cuenta la necesidad de indagar sobre la situación actual de la comunidad objetivo. No se emplea la escala de Liker o cualquier otro método, ya que no se requiere inferir sobre la percepción que tienen las mujeres cabeza de familia de la comunidad sobre una situación en particular, por lo contrario, se necesita encontrar datos que nos lleven a visualizar cual es la real situación de estas, en cuanto a sus condiciones de vida, y necesidades fisiológicas y sociales.

El siguiente esquema describe la estrategia de responsabilidad social empresarial asociada al modelo diseñado para comunidades asentadas en un entorno empresarial específicamente para mujeres cabeza de familia, el cual plantea la satisfacción de necesidades sociales básicas, de educación y económicas a partir del sistema de donación de mercancías, operacionalizado en tres programas como son el de Recursos Educativos, el de Beneficio Económico y el Social, proyectando a partir de éstos un acercamiento de las empresas al grupo objetivo, propendiendo por el mejoramiento de la calidad de vida del mismo. 


\section{FIGURA 1. ESQUEMA DE MODELO DE RSE CASO MUJERES CABEZA DE FAMILIA.}

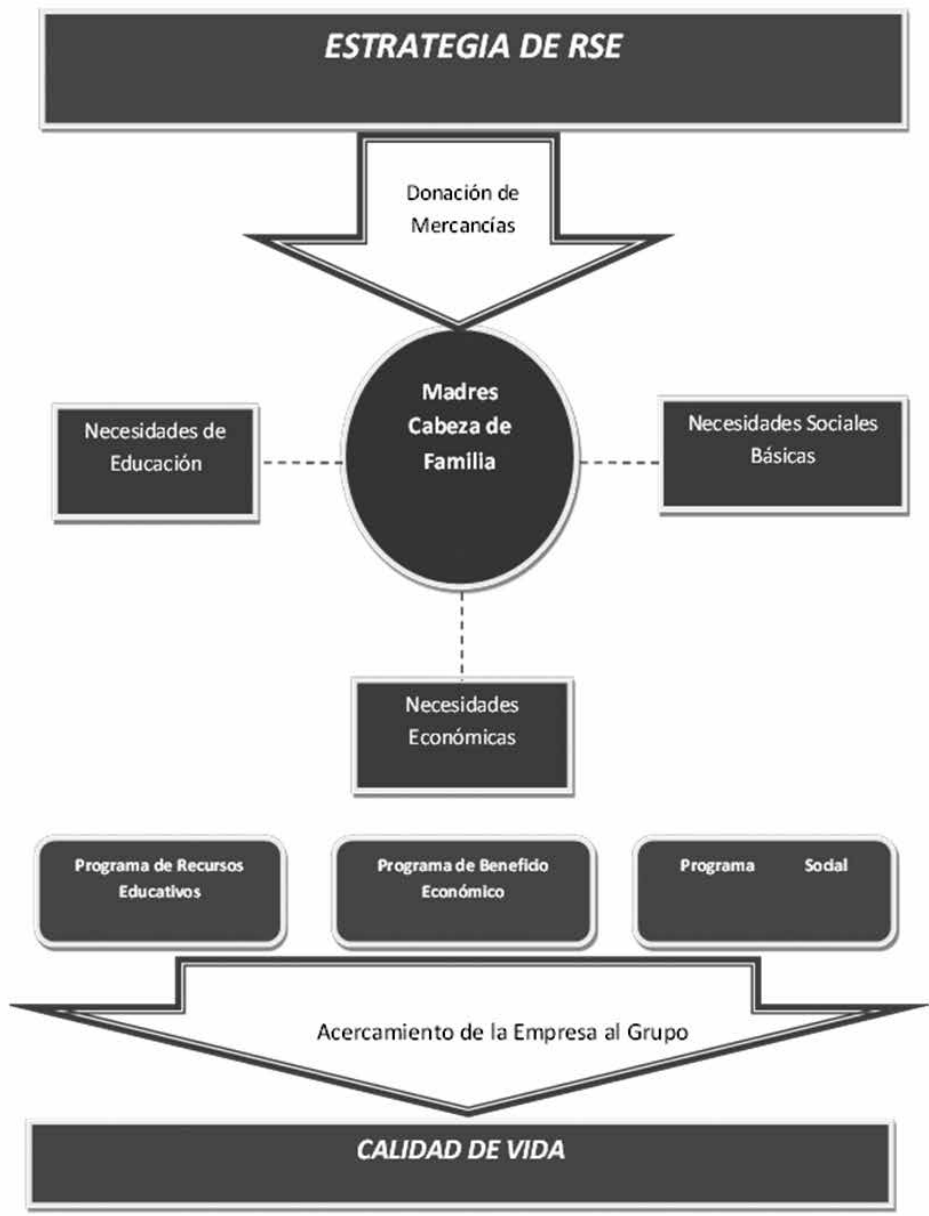

Fuente: (7)

\section{INTERPRETACIÓN DE LOS DATOS}

Lo reflejado en las encuestas validadas, muestra que para el $42,5 \%$ de las mujeres cabeza de familia sus ingresos oscilan entre \$ 200.001 y los \$300.000; solamente el 7,5\% muestra ingresos superiores a los \$ 400.001 . Estas cifras reflejan que el $92,5 \%$ de estas mujeres tienen ingresos por debajo del salario mínimo.

El 37,5\% de las encuestadas expresa que sus hogares están compuestos por entre 1 y 3 miembros. El 57,5\% de los hogares son conformados entre cuatro y siete miembros, y el porcentje restante por más de siete miembros. 
En cuanto a miembros del hogar dependientes de estas mujeres, el $45 \%$ de ellas sostiene entre cuatro y cinco, un $25 \%$ entre dos y tres, el 17,5\% entre seis y siete y el resto solo mantiene a una persona.

Se observa que ninguna de las encuestadas tiene educación profesional y solamente un $15 \%$ ha realizado estudios técnicos. Prácticamente la mitad $(42,5 \%)$, cursó hasta la secundaria y un $32,5 \%$ solo realizó estudios de primaria. El 10\% no presenta ningún tipo de formación académica.

El 10\% de las madres son menores de edad, de estas el 7,5\% tiene menos de 16 años. El 15\% son mayores de 40 años, un 17,5\% están entre los veintiuno y los veinticinco años; además el mismo porcentaje respondió estar en edades entre los treinta y uno y treinta y cinco años. El porcentaje más alto $(22,5 \%)$ se encuentra entre los treinta y seis años y los cuarenta años de edad.

Se puede ver que el $40 \%$ habita en vivienda propia, el $37,5 \%$ vive en arriendo y el 22,5\%, en casa familiar.

Se encontró que el $70 \%$ no tiene un compañero sentimental (solteras, separadas o viudas), y casi un tercio (30\%) se encuentra ligada a una relación sentimental, ya sea en estado de unión libre $(20 \%)$ o como casada $(10 \%)$.

El 20\% vive en estrato sub normal o cero (0), el $40 \%$ se encontró en el estrato 1, evidenciando así, la situación precaria en que viven. El 27,5\% vive en estrato 2 y un 12,5\% se ubica en estrato tres.

Adicionalmente, se puede observar que el $72,5 \%$ de ellas pertenecen al régimen subsidiado de salud, lo cual deja ver una alta dependencia de los programas del gobierno. Un $15 \%$ se encuentra afiliado al régimen vinculado de salud, y el 12,5\% al contributivo.

En cuanto a cifras de empleo se encontró que un $10 \%$ no trabaja, un 17,5\% solamente lo hace entre uno y tres días a la semana, el $45 \%$ trabaja entre cuatro y cinco días a la semana y el 27,5\% restante trabaja entre seis y siete días a la semana por menos de un salario mínimo. Mas del 50\% de las mujeres que trabajan, lo hacen excediendo las 8 horas de trabajo al día, legalmente establecidas, por un ingreso menor al salario mínimo, en el cual el 12,5\% trabaja una jornada desgastante de más de 10 horas al día; el $7,5 \%$ trabaja media jornada laboral, máximo seis (6) horas al día. 


\section{ANALISIS DE RESULTADOS}

Luego de la interpretación de los datos obtenidos, resultan diferentes análisis que conllevan a la determinación de los diversos programas y componentes que conforman el modelo diseñado.

En cuanto a la situación laboral, la décima parte (10\%) no trabaja. Si se tiene en cuenta que la mayor parte de estas mujeres subsisten en condiciones mínimas, con menos de un salario mínimo, se podría asegurar que es un grupo bastante vulnerable dentro del total analizado. Además el 5\% solo trabaja entre uno y dos días a la semana. Es preocupante la situación del grupo $(27,5 \%)$ que trabaja entre seis y siete días a la semana; este grupo por su tamaño es bastante considerable y se puede afirmar que en su mayoría solo consiguen ingresos por debajo de un salario mínimo y muchas de estas, aproximadamente el 37,5\% (correspondiente a más de la tercera parte) deben, entre otros gastos, pagar el arriendo en sus hogares.

Más de la mitad de la población objeto de análisis (55\%), trabaja más de ocho o diez horas diarias, lo cual es desgastante física y emocionalmente para cualquier persona. El 7,5 \% trabaja solamente media jornada laboral, con un máximo de seis horas al día. La cuarta parte restante, trabaja las ocho horas diarias que se establecen por ley, resaltando que en su mayoría solo trabajan por ingresos inferiores al salario mínimo.

Teniendo en cuenta el salario devengado, se concluye entonces que el $92,5 \%$ de las mujeres cabeza de familia, se encuentran necesitadas de apoyos económicos, ya que sus ingresos no superan un salario mínimo. Así mismo, aunque solo el 7,5\% poseen ingresos por encima de los 400.000 pesos, se observa que estas mujeres tienen una gran exigencia económica del total de su familia.

En cuanto al tema poblacional, se encontró que más de la mitad de los hogares, se encuentran conformados entre cuatro y siete miembros y un $5 \%$ está conformado por más de siete, lo que evidencia una gran exigencia económica para la mujer cabeza de familia. En conclusión, la quinta parte de los hogares, se encuentran con hacinamiento crítico con más de seis miembros en el hogar.

La alta dependencia de los miembros de la familia de las mujeres cabeza de hogar se evidencia, ya que la sexta parte $(62,5 \%)$ expresa que de ellas dependen económicamente entre cuatro y siete miembros de su familia. El 2,5 \% del total tienen a más de siete miembros a cargo, en 
su mayoría, sosteniéndose con ingresos inferiores a un salario mínimo. Adicionalmente, se identificó que más de un tercio $(37,5 \%)$ viven en condiciones de arrendamiento, lo que agrava su situación económica, ya que la mayor parte viven con menos de un salario mínimo mensual. Estas cifras coinciden con los resultados del censo del año 2005, donde se muestra que los porcentajes de mujeres cabeza de familia con vivienda propia ascienden al 54,7\%.

Las condiciones de vivienda de casi un tercio $(60 \%)$ de estas mujeres, son precarias y todas viven en estratos cero y uno. Se presentan viviendas con pisos y paredes rajadas, pisos de arena, baños en mal estado o con poca privacidad ya que no poseen puertas tanto en los baños como en los dormitorios; la mayoría de las viviendas son de madera y algunas construidas en tablas. El porcentaje de encuestadas que se ubica en estrato dos, el $27,5 \%$, también presenta condiciones precarias en la vivienda, como calles de arena, suelos de tierra y casas en malas condiciones. Se observó que en todos los estratos tanto cero, uno y dos, así como las que viven en estrato tres, se presenta una escasez de electrodomésticos básicos en la cocina como estufas y neveras, o las que los poseen se encuentran en mal estado.

En cuanto a la escolaridad, el $10 \%$ de las mujeres cabeza de familia no posee ningún tipo de estudio y el $75 \%$ solo cursó primaria o secundaria, lo que agrava su situación al momento de buscar empleo, ya que no cuentan con ninguna capacitación básica para el trabajo. Solo un $15 \%$ de mujeres cabeza de familia se tienen un grado técnico, resaltando a una de ellas que cuenta con este grado y tiene menos de veinte años. Se destaca el hecho que en la muestra no se encontró ninguna persona con grado de profesional. Con relación a las edades de las mujeres, se resalta que el 10\% son madres adolescentes y un 17,5 \% madres menores de veinte años. El 15\% del total, se encuentran en edades por encima de los cuarenta años, lo que sumado al grado de escolaridad repercute en su situación de empleo, ya que son pocas las empresas que contratan personas por encima de esta edad.

El $70 \%$ no tiene un compañero estable, ya que se encuentran solteras, viudas o separadas. Más de la mitad son solteras (52,5\%) y el $30 \%$ vive en unión libre o están casadas, teniendo la ayuda de un compañero para la crianza o cuidado de los hijos y personas a cargo. Adicionalmente, se pudo encontrar que en un gran porcentaje de los hogares visitados, los niños se encontraban solos con adultos mayores. Esto se puede contrastar con la situación laboral, ya que el $82 \%$ trabaja mínimo las 8 horas establecidas por ley. 
Aproximadamente, tres cuartas partes, que representan un $72,5 \%$ se encuentran amparadas bajo el régimen subsidiado de salud, que por definición es aquel donde el gobierno aporta un subsidio a la salud de las personas menos favorecidas (pobres), entre ellas mujeres en embarazo o cabezas de familia cubriendo las necesidades básicas en salud (8), lo cual se asemeja al valor nacional que según datos de Economía Colombiana (2011), el 70\% de la población colombiana hace parte de este régimen.

Con relación al régimen contributivo de salud, se encontró que el 12,5\% se encuentran en el régimen, es decir, un poco más de la decima parte solamente. A este régimen pertenecen todas aquellas personas que devenguen un sueldo, los cuales aportan una determinada cantidad de dinero a una EPS para la prestación de los servicios de salud, de él y de su grupo familiar. En este se encuentran los trabajadores independientes y dependientes, los pensionados y demás que devenguen un salario (Suaza, 2006).

Adicionalmente, se encontró que un $15 \%$ pertenecen al régimen vinculado de salud, lo cual es un indicador preocupante, ya que solo reciben medicina por urgencias. Este es un régimen temporal, en el cual están registradas todas aquellas personas que no tienen ningún tipo de cobertura en salud, por lo que se tienen en cuenta para la afiliación en el régimen subsidiado, pero a su vez presta los servicios básicos en salud.

\section{MODELO DE RSE APLICADO A COMUNIDADES: CASO MUJERES CABEZA DE FAMILIA.}

De acuerdo con el análisis de los resultados obtenidos de la aplicación de los instrumentos a la comunidad y en especial a las mujeres cabeza de familia se pudo definir y delimitar los diferentes elementos, procesos y relaciones que presenta el modelo definido. Este modelo fue diseñado de manera gráfica con unas interacciones lógicas y secuenciales que serán motivo de explicación.

Las dimensiones que se observan en el modelo, están basadas en la pirámide de la responsabilidad social de la empresa de Carroll (9) y en el enfoque filantrópico de la RSE, el cual citando a Quazi y O'Brien (10) dibuja una visión amplia de la RSE en la cual las empresas están dispuestas a realizar donaciones, incluso aunque estas se perciban como un costo neto para la empresa. Las donaciones pueden ser en dinero o en especie, con un carácter gratuito, donde no hay recuperación de la inversión, ni se controla lo que el donatario hace con el aporte donado. Sin embargo, para 
efectos de seguimiento, y como requisito del modelo, se ha implementado unos indicadores para poder medir los esfuerzos en materia de RSE y el impacto del modelo en la calidad de vida del grupo objetivo.

Como una forma de operacionalización de este modelo se diseñaron tres programas, alineados con las dimensiones definidas, el Programa Social, el Programa de Recursos Económicos y el Programa de Recursos Educativos, los cuales propenden por la mejora en la calidad de vida del grupo objeto de estudio y el acercamiento de la empresa a las mujeres cabeza de familia de la comunidad; todo esto teniendo en cuenta las variables que se observan en el entorno y que afectan al grupo objetivo, alineando los elementos para minimizar su impacto.

En cuanto al modelo diseñado, el bloque de la Misión Institucional e Imagen Corporativa, se convierte entonces en el primer bloque del modelo ya que interactúa inicialmente con la Dimensión Social del cual es proveedor, ya que permite soportar estas acciones de RSE. La empresa debe, desde su gestión integrar lo social y lo económico (11) incorporando la estrategia de RSE a todos los objetivos de la empresa (12)

\section{FIGURA 2. MODELO DE RSE APLICADO A COMUNIDADES: CASO MUJERES CABEZA DE FAMILIA.}

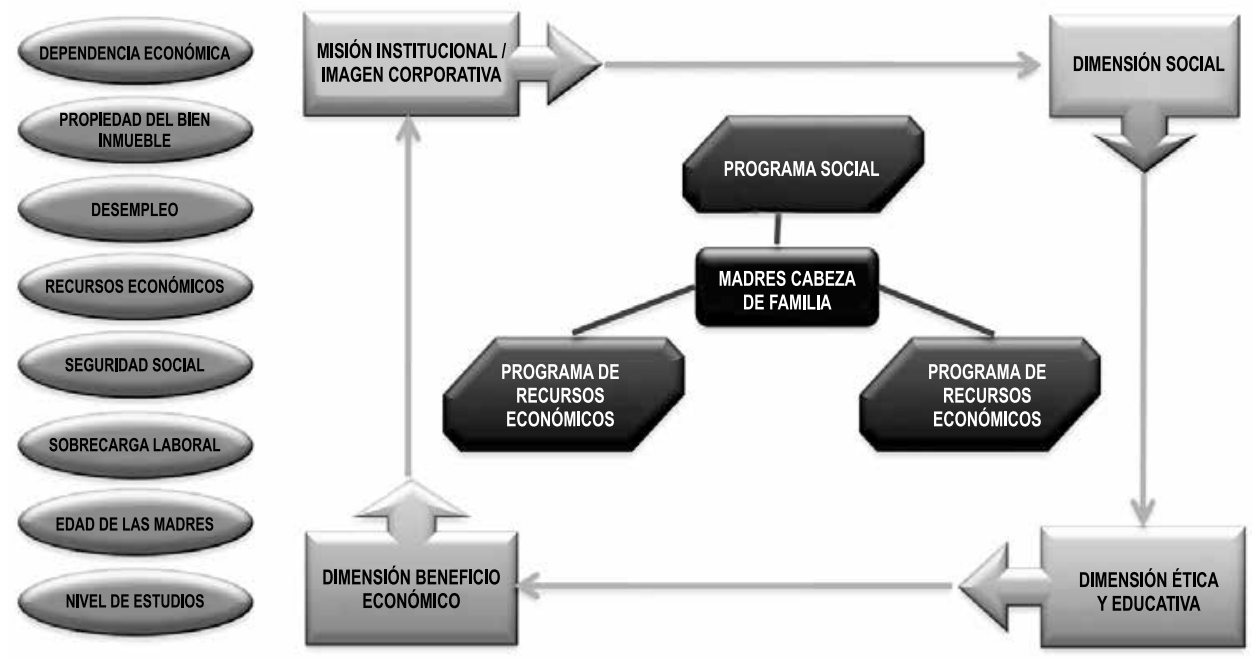

Fuente: Henríquez, G., Puche, R. 2011.

En la Dimensión Social, se llevará a cabo el Programa Social que busca satisfacer las necesidades básicas y promover estilos de vida saludables. Para ello se proponen diferentes estrategias como son: apoyar los 
programas de planificación familiar de las entidades gubernamentales por medio de la donación de métodos anticonceptivos, con el fin de evitar embarazos no deseados, la proliferación del problema de madres adolescentes y el hacinamiento crítico de los hogares, solicitando a dichas entidades la incorporación a estos programas de las mujeres cabeza de familia. Direccionar en mayor cantidad las ayudas y donaciones, a las mujeres cabeza de hogar desempleadas y aquellas con un mayor número de miembros a cargo en el hogar. Realizar donaciones de juguetes a las mujeres cabeza de familia, en cuyos hogares hallan menores de edad. De igual forma, llevar a cabo brigadas o programas especiales de donaciones de juguetes en fechas especiales como navidad, día del niño, día de los angelitos. Con el propósito de mejorar condiciones de salubridad e higiene en baños y cocinas, se sugieren donaciones de detergentes e implementos de aseo para el hogar, ya que se observaron condiciones de poca higiene y asepsia en baños y cocinas.

Posterior a la Dimensión Social, continúa la Dimensión Ética y Educación, donde se busca contribuir con un mejor nivel de estudio entre las mujeres cabeza de familia beneficiadas y sus hijos o personas a cargo. En esta dimensión se encuentra el Programa de Recursos Educativos, en el cual se pretende aportar los elementos necesarios para el desarrollo de estudios al grupo objetivo y a sus miembros del hogar. Entre otras recomendaciones para desarrollar este programa se encuentra la donación de mercancías como libros y revistas, lápices, bolígrafos, borradores, maletines y demás implementos, útiles y herramientas para adelantar estudios. Canalizar las ayudas educativas preferiblemente en temporada escolar, efectuando donaciones de artículos e implementos escolares para los menores a cargo del grupo objeto de estudio. Realizar un mayor número de donaciones consistentes en computadores, entre el grupo de mujeres cabeza de familia que quieran adelantar estudios superiores, o técnicos, dándole mayor cabida aquellas que hayan cursado por lo menos el noveno grado de bachillerato, ya que pueden desarrollar programas de formación para el trabajo con entidades como el SENA.

En cuanto a la parte Ética de esta dimensión, la satisfacción de ayudar en la mejor formación de personas para el bienestar de la sociedad, con el propósito de combatir la corrupción es una de las premisas en este modelo. Para ello se recomienda realizar seguimiento y labores de control entre las mujeres beneficiadas con el modelo, para evitar que las mercancías donadas a estas, tengan una destinación específica, es decir, que sean vendidas o entregadas a terceros. 
La interacción siguiente es entre la Dimensión Ética y Educación, con la Dimensión de Beneficio Económico, la cual busca generar beneficios para el grupo objeto de aplicación del modelo. La interacción básica entre estas dos dimensiones, obedece a la forma trasparente como se destinan los recursos para la implementación del modelo.

En esta dimensión, se encuentra el Programa de Beneficio Económico, que responde a todos los beneficios que se llevan a la comunidad objetivo para la satisfacción de algunas de sus necesidades fisiológicas básicas como por ejemplo, la alimentación y el vestir, mediante la donación de alimentos y mercancías perecederas, además de ropa. Se busca un ahorro económico a las mujeres cabeza de familia, ya que no necesitarán invertir en los rubros de las mercancías donadas.

En esta dimensión se busca extender el campo de acción hacia las personas dependientes de estas mujeres cabeza de familia, con el fin de ayudar con la variable de la dependencia económica y disminuir así, la carga económica del grupo objeto de estudio. Al disminuir la carga económica, se pretende ayudar con una mejor disposición y administración de recursos económicos para la mujer cabeza de familia, variable que también hace parte del estudio de esta dimensión. En esta variable (recursos económicos), se proyecta la contribución del modelo por medio del ahorro significativo que representa la donación de mercancías no solo para las mujeres cabeza de familia, sino, de su núcleo familiar en general.

Se pretende mejorar la calidad de vida de los beneficiarios en esta dimensión, favoreciendo en mayor proporción aquellas mujeres cabeza de familia que trabajen menos días a la semana, o se encuentren desempleadas, teniendo así, menor capacidad de generación de ingresos. En cuanto a la variable de propiedad de bien inmueble, se quiere minimizar su impacto por medio de la priorización del grupo sin vivienda propia, o peores condiciones de la vivienda.

Entre las recomendaciones para operacionalizar este programa se encuentra el direccionar en mayor cantidad la donación de alimentos y bienes perecederos que satisfagan las necesidades primarias del grupo objetivo, ya que la mayor parte de las mujeres analizadas tienen ingresos superiores a los cuatrocientos mil pesos mensuales. Con esto se busca mejorar las condiciones mínimas de vida de los beneficiados con el modelo. Priorizar las donaciones de electrodomésticos consistentes en estufas, lavadoras, neveras y otros que mejoren las condiciones de vida de aquellas mujeres cabeza de familia, cuyos hogares carezcan de estos, o, 
se encuentren en condiciones deficientes. Donar mayor cantidad de ropa a los hogares más numerosos conformados por más de 4 miembros. Dar prioridad, al conjunto de mujeres cabeza de familia que se encuentran viviendo en condición de arriendo, y aquellas que viven en casas de familias. Así mismo, llevar a cabo donaciones con mayor frecuencia, entre las mujeres cabeza de familia que se encuentran solteras, viudas o separadas, y aquellas que viven en los estratos cero y uno.

Aunque el modelo no es circular, se ha querido plantear de forma tal que muestre la intención de un ciclo de mejoramiento continuo, el cual se retroalimenta al final de todo el proceso, es decir, entre la dimensión tres y la uno, para volver a comenzar una vez se realiza otra donación de mercancía; corrigiendo en ésta, los errores que se identifiquen en la anterior donación. De esta manera, se puede proponer el grupo de mejoras que permitan abarcar un mayor número de personas beneficiadas y ser más eficientes y eficaces en la repartición de las mercancías, para llevar así, más bienestar social y económico a más colombianos, en este caso, a un mayor número de mujeres cabeza de familia.

Además se debe tener en cuenta otras recomendaciones para el mejoramiento del modelo y la posible inclusión de nuevos actores a este. Estas recomendaciones se orientan hacia la socialización de este modelo de gestión en aras de incorporar a otras organizaciones, empresas, e instituciones del sector para que se vinculen a esta causa con el fin de llevar a cabo programas de responsabilidad social empresarial creando sinergias con estas organizaciones, para que complementen el modelo. También, la donación de mercancías se debe realizar de la mano de los directivos y con el uso de piezas publicitarias de la entidad, con el fin de reforzar su imagen y generar un impacto entre el grupo objetivo.

Finalmente, los indicadores propuestos para evaluar el modelo son los siguientes:

\begin{tabular}{|c|c|c|c|c|c|}
\hline \multirow{2}{*}{$\begin{array}{c}\text { Total de Donaciones } \\
\text { Orientadas a la Comunidad } \\
\text { Objetivo } \\
\end{array}$} & \multirow[b]{2}{*}{ es igual a: } & Donaciones de Mercancias a la Comunidad Objetivo en e & laño & \multirow[b]{2}{*}{$x$} & \multirow[b]{2}{*}{100} \\
\hline & & \multicolumn{2}{|l|}{ Total de Donaciones Real izadas en el año } & & \\
\hline \multirow{2}{*}{$\begin{array}{c}\text { Indice de Personas } \\
\text { Beneficiadas }\end{array}$} & \multirow{2}{*}{ es igual a: } & Total de Personas Beneficiadas & & \multirow{2}{*}{$x$} & \multirow{2}{*}{100} \\
\hline & & Total de Personas Pertenecientes a la Comunidad Obje & & & \\
\hline \multirow[b]{2}{*}{$\begin{array}{c}\text { Indice de Crecimiento de las } \\
\text { donaciones }\end{array}$} & \multirow{2}{*}{ es igual a: } & Total de Donaciones efectuadas en el año (en pesos ) & \multirow[b]{2}{*}{-1} & \multirow[b]{2}{*}{$\mathrm{x}$} & \multirow{2}{*}{100} \\
\hline & & I de Donaciones efectuadas en el año anterior (en pesos) & & & \\
\hline
\end{tabular}


Al evaluar las donaciones realizadas en el año, se evidenciará la regularidad de la aplicación del modelo en la empresa. El segundo indicador refleja la cantidad de personas de la comunidad objetivo beneficiadas y finalmente se analiza el crecimiento de las donaciones con el tiempo lo cual finalmente determinará el acercamiento de la empresa al grupo objetivo, repercutiendo en el mejoramiento de su calidad de vida.

\section{CONCLUSIONES}

El objetivo principal de este trabajo se logró diseñando el modelo de RSE para el mejoramiento de comunidades asentadas en un entorno empresarial enfocándose en mujeres cabeza de familia, aplicando a dicho grupo las variables más críticas identificadas como incidentes en las mujeres cabeza de familia. Estas variables que fueron motivo de estudio a lo largo del trabajo se tuvieron en cuenta al momento de diseñar el modelo, indicando las más críticas con el fin de minimizar el impacto negativo de estas en el grupo objetivo del trabajo.

Se describieron las distintas variables que se observaron en el entorno y que afectan a las mujeres cabeza de familia, logrando además la identificación del grupo de interés más vulnerable para un entorno empresarial.

Finalmente, para la operacionalización del modelo se diseñaron tres programas en lo social, beneficio económico y de recursos educativos que mejoran la calidad de vida y permiten el acercamiento de la entidad al grupo objeto de estudio, enmarcados en tres dimensiones como son la Dimensión Social, Dimensión Ética y de Educación y Dimensión de Beneficio Económico.

\section{REFERENCIAS BIBLIOGRÁFICAS}

1. Angulo, Alejandro y Velásquez, Sandra. Estudios post-censales 10, DANE. [En línea] [Citado el: 11 de Octubre de 2010.] www.dane.gov.o.

2. Fuentes, Lya. El origen de una política. Mujeres jefas de hogar en Colombia, 1990-1998. Bogotá: Arfo, 2002.

3. DANE. Encuesta nacional de hogares. [En línea] 2008. www.dane. gov.co.

4. Carvajal, Manuel. Acciones pensando en la Responsabilidad Social, no siempre son rentables. 39, México: Economía sociedad y territorio, 2007, Vol. 4. JEL: M21. 
5. Barrios, Helena María. Organizaciones, análisis organizacional, aprendizaje organizacional, gestión del conocimiento. 2009.

6. López, Andrés. La sociedad y la Responsabilidad Social Corporativa como modelo de progreso. s.l.: American Androgogy University, 2009.

7. Puche, Ricardo y Henríquez, Gustavo. Diseño de modelo de responsabilidad social que permita el mejoramiento de la calidad de vida de las mujeres cabeza de familia del barrio Rebolo de Barranquilla. Barranquilla: s.n., 2011.

8. Suaza, Luis. Universidad de Antioquia. [En línea] Septiembre de 2006. www.aprendeenlinea.udea.edu.co.

9. Carroll, Archie. The pyramid of corporate social responsibility: Toward the moral management of organizational stakeholders. 4, Indiana: Elsvier, 1991, Vol. 34. JEL: M14. DOI: 10.1016/0007-6813 (91) 90005-G.

10. Quazi, A.M. y O'Brien, D. 1, s.l.: An Empirical Test of cross National Model of corporate social responsability. Journal business ethics, 2000, Vol. 25. JEL: M14.

11. Balaguer, María, Fernández, María y Muñoz, María. La responsabilidad social de la empresa. Valencia: Generalitat, 2007. ISBN: 978-84-4824699-0.

12. Smith, Craig. Corporate social responsibility: not whether, but how? No. 03-701, London: London Business School, 2003. JEL: M14.

13. Economía Colombiana. Sistemas POS en Colombia. [En línea] 2011. www.encolombia.com/economia.

14. Carroll, Archie. The pyramid of corporate social responsibility: Toward the moral management of organizational stakeholders. 4, Indiana: Elsvier, 1991, Vol. 34. DOI: 10.1016/0007-6813(91)90005-G. 